\title{
HIV-assoziierte Malignome
}

\author{
M. Hentrich ${ }^{a} \quad$ H. Jäger ${ }^{b} \quad$ R. Hartenstein ${ }^{a}$ \\ ${ }^{\text {a }}$ Städtisches Krankenhaus München-Harlaching, IV. Medizinische Abteilung, Akademisches Lehrkrankenhaus der Unversität und \\ ${ }^{b}$ Kuratorium für Immunschwäche, München
}

\section{Schlüsselwörter}

Kaposi-Sarkom · HIV-assoziiertes Non-Hodgkin-Lymphom · HIV-assoziiertes Zervixkarzinom · HIV-assoziierte Malignome $\cdot$ Nicht-AIDS-definierende Malignome

\section{Zusammenfassung}

$\mathrm{Zu}$ den AIDS-definierenden Malignomen zählen das Kaposi-Sarkom, Non-Hodgkin-Lymphome (NHL) und das invasive Zervixkarzinom. In den letzten Jahren wurde eine Zunahme des Kaposi-Sarkoms als AIDS-Sekundärmanifestation beobachtet. Pathogenetisch kommt dem humanen Herpesvirus- 8 die Bedeutung eines infektiösen Kofaktors zu. Das therapeutische Vorgehen sollte sich an der individuellen Situation und an prognostischen Faktoren orientieren. Neben lokalen Therapieverfahren stehen als systemische Behandlung Interferon- $\alpha$ und verschiedene Zytostatika zur Verfügung, wobei liposomale Anthrazykline bei fortgeschrittener Erkrankung besondere Vorteile aufweisen. Es ist anzunehmen, daß die Inzidenz HIV-assoziierter NHL wegen längerer Krankheitsverläufe bei antiretroviraler Behandlung weiter zunehmen wird. Im Regelfall sind intensivierte Chemotherapien Standardprotokollen wie $\mathrm{CHOP}$ gegenüber nicht überlegen. Etwa die Hälfte der Patienten erreicht eine komplette Remission, die Rezidivrate ist allerdings hoch. Eine optimale supportive und antiretrovirale Behandlung gehört wegen der Gefahr opportunistischer Infektionen zum Behandlungskonzept. Das invasive Zervixkarzinom stellt in urbanen Risiko-Populationen zum Teil das häufigste AIDS-definierende Malignom der Frauen dar. Die Erkrankung wird in vielen Fällen in fortgeschrittenen Stadien entdeckt und zeigt einen aggressiven Verlauf. Verschiedene andere maligne Erkrankungen, wie z.B. das Analkarzinom, der Morbus Hodgkin oder Seminome bei Patienten mit AIDS, treten im Rahmen der HIV-Infektion gehäuft auf.

\section{Key Words}

Kaposi's sarcoma · HIV-associated Non-Hodgkin's lymphoma · HIV-associated invasive cervical cancer · HIVassociated malignancies · Non-AIDS-defining malignancies

\section{Summary}

Kaposi's sarcoma (KS), Non-Hodgkin's lymphma (NHL) and invasive cervical cancer are considered AIDS-defining malignancies. The incidence of Kaposi's sarcoma has recently been increased as a secondary manifestation of AIDS. The pathogenesis is not completely understood. Human herpesvirus 8 could be identified as an infectious cofactor. Therapeutic strategies should be based on prognostic factors and tailored to the patient's individual situation. Local treatments widely used are cryotherapy or radiotherapy. Systemic therapies such as interferon- $\alpha$ or single and multiagent chemotherapy are also well established. Based on their high response rates and favorable toxicity profile, liposome-encapsulated anthracyclines may be considered first-line therapy for advanced AIDS-related KS. HIV-associated NHL may increase in frequency as HIV-infected individuals survive longer with improved antiretroviral therapy. There is no advantage for intensive as compared with standard or less intensive chemotherapeutic regimens of the $\mathrm{CHOP}$ type. Complete remissions can be achieved in approximately $50 \%$ of the patients, but the recurrence rate is high. The therapeutic strategy should include an optimal supportive care and antiretroviral treatment because chemotherapy significantly increases the risk of opportunistic infection. In urban populations at risk, cervical cancer is a common AIDS-related malignancy in women. Patients with cervical carcinoma usually have a more aggressive and more advanced disease. Various malignant diseases, such as Hodgkin's lymphoma, anal cancer or seminoma in patients with AIDS occur at higher frequency in HIV infection.

\begin{tabular}{ll}
\hline KARGER & @ 1999 S. Karger GmbH, Freiburg \\
Fax +49 7614520714 & Accessible online at: \\
$\begin{array}{l}\text { E-mail kargergmbh@aol.com } \\
\text { www.karger.com }\end{array}$ & http://BioMedNet.com/karger
\end{tabular}

Dr. Marcus Hentrich

Städtisches Krankenhaus München-Harlaching

Akademisches Lehrkrankenhaus der Universität München

IV. Medizinische Abteilung

Sanatoriumsplatz 2, D-81545 München (Germany)

Tel. +49 89 6210-2731, Fax -2443 


\section{HIV-assoziierte Malignome}

In Deutschland muß mit jährlich etwa 2000-2500 HIV-Neuinfektionen gerechnet werden. Obwohl wegen der Verfügbarkeit einer hochwirksamen antiretroviralen Therapie (HAART, highly active antiretroviral treatment) und anderer prophylaktischer Maßnahmen die Zahl neuer AIDS-Fälle auf rund 900 pro Jahr zurückgegangen ist, muß wegen längerer Krankheitsverläufe mit einer Zunahme HIV-assoziierter maligner Erkrankungen gerechnet werden.

\section{Kaposi-Sarkom}

\section{Epidemiologie, Pathogenese, Prognose}

Das Kaposi-Sarkom (KS) ist das häufigste AIDS-definierende Malignom. Während Prävalenz und Inzidenz in den letzten Jahren in etwa gleich blieben, ging die Häufigkeit als AIDSIndikator-Erkrankung seit Anfang der achtziger Jahre von $30-40 \%$ auf $10-15 \%$ in den letzten Jahren zurück.

Die Pathogenese des KS ist noch nicht vollständig geklärt. Histologisch handelt es sich um einen Spindelzelltumor vermutlich endothelialer Herkunft mit aberranter Proliferation vaskulärer Strukturen. Das humane Herpesvirus-8 bzw. KaposiSarkom-assoziierte Herpesvirus (HHV8/KSHV) wird als infektiöser Kofaktor in mehr als $90 \%$ aller, nicht nur der HIVassoziierten, Kaposi-Läsionen gefunden. $\mathrm{Zu}$ den zahlreichen weiteren pathogenetischen Faktoren zählen eine ausgeprägte Zytokin-Dysregulation mit autokriner und parakriner Stimulation des KS-Zellwachstums sowie eine Wachstumsstimulation bzw. Apoptosehemmung durch die Produkte des HIV-tatGens und des HIV-bcl2-Gens [1, 2]. Das Kaposi-Sarkom ist eine systemische, meist multifokal auftretende Erkrankung. Das von der AIDS Clinical Trial Group bereits 1989 vorgestellte Staging-System definiert zwei prognostisch unterschiedliche Gruppen (Tab. 1). Diese Einteilung hat sich im wesentlichen bewährt, allerdings scheint die Tumorlast nur bei CD4-Zellzahlen $<200 / \mu l$ prognostisch relevant zu sein [3].

\section{Therapie}

Die Therapie ist palliativ. Die Wahl der Behandlung sollte individuell auch unter Beachtung kosmetischer Aspekte erfolgen und sich an den jeweiligen prognostischen Faktoren orientieren. Eine eindeutige Lebensverlängerung durch therapeutische Maßnahmen ist bisher nicht belegt, in Einzelfällen (z.B. bei pulmonalem Befall) aber wahrscheinlich. Seit der breiten Verfügbarkeit einer HAART mehren sich Beobachtungen und Berichte über Remissionen unter alleiniger antiretroviraler Behandlung.

\section{Lokale Therapieverfahren}

Wegen des systemischen Charakters der Erkrankung sollten lokale Therapieverfahren Situationen vorbehalten sein, die durch eine begrenzte Tumormanifestation mit lokalen, die Lebensqualität beeinträchtigenden Läsionen bei insgesamt geringer Krankheitsdynamik gekennzeichnet sind. Auch bei Patienten mit fortgeschrittener Erkrankung und schlechtem Allgemeinzustand kann eine lokale Behandlung hilfreich sein.

Unter der Vielzahl der lokalen Behandlungsmodalitäten kommt der Strahlentherapie wegen hoher Ansprechraten von $80-90 \%$ besondere Bedeutung zu. Kosmetisch belastende Läsionen bilden sich ohne wesentliche systemische Nebenwirkungen zurück. Lokal wirksam sind auch kryotherapeutische Eingriffe (bei Läsionen $<0,5 \mathrm{~cm}$ ) sowie, bei Läsionen von maximal $2 \mathrm{~cm}$, intraläsionale (und meist schmerzhafte) Gaben von Vinca-Alkaloiden oder HCG [1, 2]. Diese Methoden erfordern viel Erfahrung in ihrer Anwendung.

\section{Systemische Therapie}

Bei progredienter Erkrankung oder solcher mit hoher Tumorlast können schnelle Remissionen nur durch eine systemische Behandlung erzielt werden. An etablierten Verfahren steht die Gabe von Interferon- $\alpha$ (IFN- $\alpha$ ) oder die Verabreichung einer systemischen Chemotherapie zur Verfügung. Fester Bestandteil eines systemischen Therapiekonzeptes sollte in jedem Fall eine HAART sein. Die prognostische Relevanz (Endpunkt: Überleben) einer Viruslastreduktion ist bei HIVinfizierten Patienten ohne maligne Erkrankung mittlerweile vielfach belegt.

\section{Interferon}

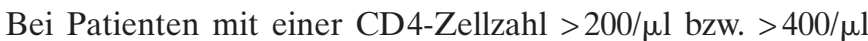
führt IFN- $\alpha$ in zum Teil hohen Dosierungen (bis zu 36 Mio IE pro Tag) zu Remissionsraten (RR) von 30 bis maximal 50\%. Problematisch ist hierbei das Nebenwirkungsprofil. Im Falle früherer opportunistischer Infektionen oder bei CD4-Zellzahlen $<200 / \mu$ l sind die Ansprechraten deutlich geringer (unter 10\%). Dauerhafte Remissionen sind selten. Durch die Kombination von IFN- $\alpha$ (4,5-18 Mio IE pro Tag) und Zidovudin konnten die Remissionraten auch bei Patienten mit CD4-Zellzahlen $<200 / \mu l$ gesteigert werden (RR 20-30\%) [4]. Studien zur Kombination von IFN- $\alpha$ mit Proteasehemmern laufen derzeit. Patienten mit pulmonalem Befall oder rasch progredienter Erkrankung sollten wegen des oft protrahierten Wirkungseintritts (Wochen bis Monate) nicht mit IFN- $\alpha$ behandelt werden.

Chemotherapie

Schnelle Remissionen werden nur durch eine zytostatische Chemotherapie induziert. Je nach Patientenselektion führen Monotherapien mit Bleomycin, Vinca-Alkaloiden, Etoposid oder Doxorubicin zu Ansprechraten von etwa 25 bis maximal $70 \%[1,2]$. Die Ansprechraten der Chemotherapie-Kombinationen liegen etwas höher, allerdings auf Kosten einer teilweise gesteigerten Toxizität. Die Remissionsdauer beträgt etwa 5-9 Monate. Als relativ gut verträglich erwies sich die Kombination von niedrigdosiertem Doxorubicin, Bleomycin und Vincristin (ABV), appliziert in 14tägigen Intervallen (RR 60-88\%). Liposomale Anthrazykline haben die Therapie des Kaposi-Sarkoms entscheidend bereichert und werden derzeit als Chemotherapie der ersten Wahl betrachtet. Pegyliertes liposomales Doxorubicin (Caelyx ${ }^{\circledR}$, Essex Pharma, München) führte in 3 Phase-III-Studien zu relativ hohen Remissionsraten (46-79\%) bei insgesamt günstigem Toxizitätsprofil $[5,6]$. Liposomales Daunorubicin (DaunoXome ${ }^{\circledR}$, Nexstar Pharmaceuticals, Martinsried) erwies sich im randomisierten Vergleich gegenüber ABV als äquieffektiv (RR 25 verus 28\%), war jedoch mit geringerer Neurotoxizität und Alopezierate verbunden [7]. Bei Patienten mit fortgeschrittenen und chemo- 
Tab. 1. Klassifikation des HIV-assoziierten Kaposi-Sarkoms (nach ACTG)

\begin{tabular}{|c|c|c|}
\hline & Frühstadium (good risk) & Spätstadium (poor risk) \\
\hline \multirow[t]{2}{*}{ Tumor (T0-T1) } & $\begin{array}{l}\text { begrenzt auf Haut, Lymphknoten, } \\
\text { minimale orale Beteiligung } \\
\text { (keine erhabenen Läsionen) }\end{array}$ & $\begin{array}{l}\text { viszeraler Befall, } \\
\text { ausgedehnte orale Beteiligung, } \\
\text { tumorbedingte Ödeme oder Ulzerationen }\end{array}$ \\
\hline & und & oder \\
\hline \multirow[t]{2}{*}{ Immunsystem (I0-I1) } & CD4-Zellzahl > 200/ $\mu \mathrm{l}$ & CD4-Zellzahl <200/ $\mu$ l \\
\hline & und & oder \\
\hline $\begin{array}{l}\text { Systemische (S0-S1) } \\
\text { Erkrankung }\end{array}$ & $\begin{array}{l}\text { keine opportunistischen Infektionen, } \\
\text { keine oropharyngeale Candidiasis, } \\
\text { Karnofsky-Index } \geq 70, \\
\text { keine B-Symptomatik }^{\mathrm{a}}\end{array}$ & $\begin{array}{l}\text { opportunistische Infektionen, } \\
\text { oropharyngeale Candidiasis, } \\
\text { Karnofsky-Index }<70 \text {, } \\
\text { B-Symptomatik }{ }^{\mathrm{a}} \text {, } \\
\text { andere HIV-assoziierte Erkrankungen }\end{array}$ \\
\hline
\end{tabular}

therapeutisch vorbehandelten KS erwiesen sich in neueren Arbeiten Vinorelbin (RR 33\%) und Paclitaxel (RR 50-70\%) als wirksam [8].

Unter einer HAART ist in vielen Fällen der Versuch einer Spreizung der Zyklusintervalle oder einer Pause der Chemotherapie gerechtfertigt.

\section{Non-Hodgkin-Lymphome}

\section{Epidemiologie und Pathogenese}

Ungefähr 3\% aller HIV-infizierten Personen und bis zu 29\% der Patienten mit fortgeschrittener HIV-Infektion erkranken an einem Non-Hodgkin-Lymphom (NHL). Es handelt sich zu etwa $80 \%$ um hochmaligne B-Zell-Lymphome. Als häufigste Form, insbesondere bei fortgeschrittener Immundefizienz, tritt das diffus großzellige B-Zell-Lymphom auf. Als neuer Subtyp dieser Entität wurde bei HIV-infizierten Patienten das plasmablastische Lymphom beschrieben [9]. 20-30\% betreffen Burkittlike NHL, bis zu 20\% der hochmalignen Formen sind nicht exakt klassifizierbar. Eine relativ seltene Besonderheit stellt das HHV8- und EBV-assoziierte «primary effusion lymphoma» dar, das sich ausschließlich in Form von Körperhöhlenergüssen manifestiert [10].

HIV-NHL geht in über einem Drittel der Fälle eine Lymphadenopathie voraus.

Pathogenetisch wird eine Dysregulation verschiedener Zytokine mit chronischer Antigen-Stimulation der B-Zellen diskutiert [11]. EBV-Genom wird in Lymphomzellen bei etwa 50\% der Patienten mit HIV-NHL und bei $100 \%$ derer mit primären ZNS-Lymphomen nachgewiesen.

\section{Diagnostik und Prognose}

70-90\% der Patienten weisen bei Diagnose einen extranodalen und etwa $30 \%$ einen Knochenmarkbefall oder eine ZNS-Beteiligung auf.

Da primär ZNS-Lymphome mittels cranialer Computertomographie (CCT) oder Magnetresonanztomographie (MRT) nicht sicher von einer Toxoplasmose unterschieden werden können, wird häufig zunächst eine Toxoplasmose-Behandlung einge- leitet. Die Durchführung einer Hirnbiopsie (bei günstiger Tumorlokalisation) ist bei AIDS-Patienten nicht unproblematisch. Ein neues diagnostisches Vorgehen bietet die Bestimmung der EBV-PCR aus dem Liquor, die mit hoher Sensitivität $(80 \%)$ und Spezifität $(100 \%)$ ein zerebrales Lymphom auch ohne Hirnbiopsie zu erfassen vermag [12]. Die diagnostische Treffsicherheit dieses wenig invasiven Vorgehens wird in Kombination mit einer PET noch erhöht $[12,13]$.

Die mediane Überlebenszeit beträgt 5-7 Monate. Als prognostisch ungünstig haben sich die in Tabelle 2 dargelegten Faktoren erwiesen. Patienten, für die keines dieser Kriterien gilt, leben im Median knapp 2 Jahre. Die schlechteste Prognose weisen Patienten mit primärem ZNS-Lymphom auf (mediane Überlebensdauer: 2,5 Monate).

\section{Therapie}

Durch eine Polychemotherapie werden bei $40-70 \%$ der Patienten komplette Remissionen erreicht, die nur in Ausnahmefällen länger als 2 Jahre anhalten. Neben der hohen Rezidivrate wird die schlechte Prognose der Patienten maßgeblich durch das Auftreten opportunistischer Infektionen bestimmt. Aggressive Protokolle führen im Regelfall gegenüber Standardregimen zu keinem Überlebensvorteil [10, 15]. Im Rahmen einer Phase-III-Studie betrug die mediane Überlebensdauer unter niedrigdosiertem m-BACOD 35 Wochen im Vergleich zu 31 Wochen unter Standarddosierung des gleichen Protokolls plus GM-CSF bei höherer Toxizität des GM-CSF-unterstützten Arms [15]. Auch das CHOP-Protokoll hat sich in der Behandlung der HIV-NHL bewährt. 68\% der Patienten mit normalem (d.h. günstigen) Risiko erreichten in einer Multicenterstudie der Deutschen Studiengruppe zur Diagnose und Behandlung HIV-assoziierter NHL eine komplette Remission (CR) [16]. Hierbei ist bemerkenswert, daß unter einer Erhaltungstherapie mit Interferon- $\alpha(3 \times 5$ Mio IE pro Woche $)$ nahezu alle Patienten in CR nicht an Lymphomrezidiven, sondern an opportunistischen Infektionen verstarben. Gegenwärtig wird im Rahmen eines randomisierten Phase-III-Protokolls der Einfluß einer Erhaltungstherapie mit IFN- $\alpha$ auf das Überleben der Patienten geprüft (risikoadaptierte Induktion mit 
Tab. 2. Prognostisch ungünstige Faktoren bei HIV-assoziierten NHL

\begin{tabular}{ll}
\hline Etablierte Faktoren & $\begin{array}{l}\text { Faktoren, die in einzelnen Analysen } \\
\text { (jeweils } \mathrm{n}>90) \text { ermittelt wurden }\end{array}$ \\
\hline CD4-Zellzahl $<100 / \mu \mathrm{l}$ & LDH-Erhöhung \\
Karnofsky-Index $<70$ & Alter $>35$ Jahre \\
Vorherige AIDS-Diagnose & Stadium III/IV \\
Extranodale Manifestation & i.v. Drogen-Abusus \\
& immunoblastisches NHL \\
\hline
\end{tabular}

CHOP +/- Strahlentherapie bei dosisreduziertem Protokoll für Patienten mit ungünstigen Prognosefaktoren; nach Erreichen der CR Randomisation in einen Arm mit oder ohne IFN-a). Wegen der hohen Wahrscheinlichkeit eines zerebralen bzw. meningealen Rezidivs ist eine ZNS-Prophylaxe erforderlich. Patienten mit HIV-assoziierten NHL sollten nach Möglichkeit in diese Studie eingebracht werden (Studienleitung Prof. Huhn, Berlin, und Prof. Mitrou, Frankfurt/M.).

Wegen der mit einer Chemotherapie verbundenen Immunund Myelosuppression sind besondere Supportivmaßnahmen erforderlich [17]. Eine Primär- und Sekundärprophylaxe opportunistischer Infektionen sollte entsprechenden Richtlinien gemäß erfolgen. Hämatopoetische Wachstumsfaktoren vermindern auch bei Patienten mit HIV-NHL die Inzidenz febriler Neutropenien. Ihr Einsatz empfiehlt sich bei vorbestehender oder therapiebedingter Neutropenie. Antiretrovirale Substanzen der ersten Generation (Zidovudin, Zalcitabin) vermochten die Ergebnisse der NHL-Behandlung nicht entscheidend $\mathrm{zu}$ verbessern [14]. Die Frage, ob eine HAART parallel zur Chemotherapie eingeleitet bzw. fortgeführt werden soll, ist noch nicht geklärt. Einerseits werden ein Viruslastanstieg unter Chemotherapie sowie die Ausbildung resistenter Virusstämme durch Therapiepausen gefürchtet. Andererseits darf es durch die Myelotoxizität einer HAART nicht zur Einschränkung der Dosisintensität einer kurativen Chemotherapie kommen. Vorläufige Daten aus Italien zeigen, daß die Kombination HAART/CHOP durchführbar ist. Über einen möglichen Überlebensvorteil kann noch keine Aussage gemacht werden [18]. Fällt die Entscheidung für einen simultanen Einsatz antiretroviraler Medikamente, müssen pharmakologische Interferenzen und kumulative Myelo- und Neurotoxizitäten bedacht werden [19].

Patienten mit primärem ZNS-Lymphom stellen ein besonderes therapeutisches Problem dar wegen des meist schlechten Allgemeinzustandes mit CD4-Zellzahlen $<40 / \mu l$, einer fast $100 \%$ igen Rezidivrate und einer hohen Inzidenz opportunistischer Infektionen. Das therapeutische Vorgehen sollte sich nach dem klinischen Zustand des Patienten richten. In Einzelfällen führt eine kombinierte Radio-Chemotherapie bei Patienten in guter Verfassung und ohne meningealen bzw. spinalen Befall zu länger anhaltenden Remissionen. Auch bei gleichzeitiger peripherer Lymphom-Manifestation ist eine kombinierte Behandlung angezeigt. Mit einer alleinigen Strahlentherapie, in vielen Fällen Therapie der Wahl, können trotz einer meist kurzen Überlebenszeit gute palliative Effekte erzielt werden. Patienten mit Meningeosis sollten einer intrathekalen Behandlung (MTX oder Cytarabin), solche in sehr schlechtem Zustand alleinigen supportiven Maßnahmen zugeführt werden.

\section{Zervixkarzinom}

Das invasive Zervixkarzinom zählt seit 1993 zu den AIDSdefinierenden Malignomen und tritt bei HIV-infizierten Frauen in urbanen Risikopopulationen zum Teil häufiger auf als NHL oder das Kaposi-Sarkom [20]. HIV-assoziierte Zervixkarzinome werden meist in fortgeschritteneren Stadien diagnostiziert (Stadium II-IV in etwa drei Viertel der Fälle), zeigen ein aggressiveres Verhalten und sprechen im Regelfall auf Standardtherapien schlechter an. Die Prognose von $\mathrm{Pa}-$ tientinnen mit CD4-Zellzahlen $>500 / \mu$ l scheint etwas besser zu sein, obwohl in den meisten Fällen das Karzinom - und nicht opportunistische Infektionen - Todesursache ist. Diese Erkenntnisse beruhen im wesentlichen auf kleineren retrospektiven Serien oder Fallberichten.

\section{Nicht-AIDS-definierende HIV-assoziierte Malignome}

Einige maligne Erkrankungen treten im Rahmen der HIVInfektion gehäuft auf. Auch wenn verschiedene epidemiologische Arbeiten, die meist auf der Auswertung von Melderegistern beruhen, $\mathrm{zu}$ teils unterschiedlichen Ergebnissen kommen, ist eine erhöhte Inzidenz einiger Malignome gut belegt [21]. Das Risiko für einen Morbus Hodgkin ist bei HIV-infizierten Personen je nach Untersuchung 5-19fach, bei Patienten mit AIDS 7,6fach erhöht [21, 22]. Die Erkrankung wird im Vergleich zur Normalbevölkerung häufiger im Stadium IV und mit B-Symptomatik diagnostiziert. Histopathologische Analysen ergaben einen gesteigerten Anteil des nodulär-sklerosierenden und lymphozytenarmen Typs. Bei etwa drei Viertel der Patienten kann EBV-Genom im Lymphomgewebe nachgewiesen werden. Die Erkrankung verläuft häufig aggressiv und weist eine schlechtere Prognose auf als bei HIV-negativen Patienten (mediane Überlebensdauer: 8-15 Monate). Etwa 50\% der Todesfälle gehen auf opportunistische Infektionen zurück [22].

Mehrere epidemiologische Analysen ergaben für HIV-infizierte Männer kein erhöhtes Risiko für die Entwicklung eines Hodenkeimzelltumors. Allerdings weisen Patienten mit AIDS ein gering (2,9fach), aber signifikant erhöhtes Risiko für Seminome auf [21]. Die Prognose wird maßgeblich von der Immunsituation bestimmt. In der Regel erfolgt die jeweilige Standardtherapie unter entsprechender Supportivbehandlung problemlos [23]. Bei Patienten mit AIDS und weit fortgeschrittenen Hodentumoren sollten intensivierte Chemotherapien wegen der Gefahr opportunistischer Infektionen vermieden werden. Das Risiko für die Entwicklung eines Analkarzinoms ist bei HIV-infizierten Personen 31-62fach erhöht, wobei überwiegend homosexuelle Männer betroffen sind. Histologisch handelt es sich größtenteils um Plattenepithelkarzinome. Über Therapieergebnisse liegen nur wenige Berichte vor. Es mehren sich jedoch Hinweise darauf, daß Patienten durchaus einer kombinierten Radiochemotherapie mit Erfolg zugeführt werden können (bei auf 30 Gy reduzierter Strahlendosis). Insbesondere 
bei Patienten ohne vorherige opportunistische Infektionen wurden anhaltende Vollremissionen berichtet [24].

Seit Beginn der HIV-Epidemie sind zahlreiche weitere maligne Erkrankungen bei HIV-infizierten Personen beschrieben worden. Das Risiko, ein Basaliom zu entwickeln, ist nur bei HIV-infizierten Hämophilie-Patienten deutlich erhöht. Ein reproduzierbarer Inzidenzanstieg anderer Malignome ist bisher nicht gelungen, auch wenn vereinzelt über das gehäufte Auftreten von z. B. Bronchialkarzinomen, Plasmozytomen und Hirntumoren berichtet wurde. Allerdings scheinen viele dieser malignen Erkrankungen im Rahmen der HIV-Infektion einen aggressiveren Verlauf zu nehmen.

\section{Literatur}

1 Krown SE: Acquired immunodeficiency syndromeassociated Kaposi's sarcoma. Med Clin North Am 1997;81:471-494.

2 Jie C, Tulpule A, Zheng T, Masood R, Espina B, Gill PS: Treatment of epidemic (AIDS-related) Kaposi's sarcoma. Curr Opin Oncol 1997;9:433-439.

3 Krown SE, Testa MA, Huang J for the AIDS Clinical Trial Group Comittee: AIDS-related Kaposi's sarcoma: Prospective validation of the AIDS clinical trial group staging classification. J Clin Oncol 1997; 15:3085-3092.

4 Shepherd FA, Beaulieu R, Gelman K, Thuot CA, Sawka C, Read S, Singer J: Prospective randomized trial of two dose levels of interferon alfa with zidovudine for the treatment of Kaposi's sarcoma associated with human immunodeficiency virus infection: A Canadian HIV Clinical Trials Network study. J Clin Oncol 1998;16:1736-1742.

5 Northfelt DW, Dezube BJ, Thommes JA, Miller BJ, Fischl MA, Friedman-Kien A, Kaplan LD, DuMond Ch, Mamelok RD, Henry DH: Pegylated-liposomal doxorubicin versus doxorubicin, bleomycin, and vincristine in the treatment of AIDS-related Kaposi's sarcoma: Results of a randomized phase III trial J Clin Oncol 1998;16:2445-2451.

6 Stewart JSW, Jablonowski H, Goebel FD, L'age M, Spittle M, Opravi M, and the Doxil Trials Group: A randomized comparative trial of Doxil ${ }^{\circledR}$ versus bleomycin and vincristine (BV) in the treatment of AIDS-related Kaposi's sarcoma. Proc ASCO 1997; 16:55a(abstr 190)

7 Gill PS, Wernz J, Scadden DT, Cohen P, Mukwaya GM, von Roenn JH, Jacobs M, Kempin S, Silverberg I, Gonzales G, Rarick MU, Myers AM, Shepherd F, Sawka C, Pike MC, Ross ME: Randomized phase III trial of liposomal daunorubicin versus doxorubicin, bleomycin, and vincristine in AIDS-related Kaposi's sarcoma. J Clin Oncol 1996;14:2353-2364.
8 Welles L, Saville MW, Lietzau J, Pluda JM, Wyvill KM, Feuerstein I, Figg WD, Lush R, Odom J, Wilson WH, Fajardo MT, Humphrey RW, Feigal E Tuck D, Steinberg SM, Broder S, Yarchoan R: Phase II trial with dose titration of paclitaxel for the therapy of human immunodeficiency virus-associated Kaposi's sarcoma. J Clin Oncol 1998;16:1112-1121.

9 Delecluse HJ, Anagnostopoulos F, Dallenbach F, Hummel M, Marafioti T, Schneider U, Huhn D, Schmidt-Westhausen A, Reichart PA, Gross U, Stein H: Plasmablastic lymphomas of the oral cavity: A new entity associated with the human immunodeficiency virus infection. Blood 1997;89:1413-1420.

10 Nador RG, Ceserman E, Chadburn A, Dawson DB, Ansari MQ, Said J, Knowles DM: Primary effusion lymphoma: A distinct clinicopathologic entity associated with the Kaposi's sarcoma-associated herpes virus. Blood 1996;88:645-656

11 Straus DJ: Human immunodeficiency virus-associated lymphomas. Med Clin North Am 1997;81: 495-510

12 Cingolani A, De Luca A, Larocca LM, Amassari A, Scerrati M, Antinori A, Ortana L: Minimally invasive diagnosis of acquired immunodeficiency syndromerelated primary central nervous system lymphoma J Natl Cancer Inst 1998;90:364-369.

13 Villringer $\mathrm{K}$, Jäger $\mathrm{H}$, Dichgans $\mathrm{M}$, Ziegler $\mathrm{S}$ Poppinger J, Herz M, Kruschke C, Minoshima S, Pfister HW, Schwaiger M: Differential diagnosis of CNS lesions in AIDS patients by FDG-PET J Comput Assist Tomogr 1995;19:532-536.

14 Levine AM, Tulpule A, Espina B, Boswell W, Buckley J, Rasheed S, Stain S, Parker J, Nathwani B, Gill PS: Low dose methotrexate, bleomycin, doxorubicin, cyclophosphamide, vincristine, and dexamethasone with zalcitabine in patients with acquired immunodeficiency syndrome-related lymphoma. Cancer 1996;78:517-526.

15 Kaplan LD, Straus DJ, Testa MA, von Roenn J, Dezube BJ, Cooley TP, Herndier B, Northfelt DW, Huang J, Tulpule A, Levine A, for the National Institute of Allergy and Infectious Diseases AIDS Clinical Trials Group: Low-dose compared with standard-dose m-BACOD chemotherapy for nonHodgkin's lymphoma associated with human immunodeficiency virus infection. N Engl J Med 1997; 336:1641-1648.
16 Weiß R, Huhn D, Mitrou P, Nerl Ch, Schürmann D, Scheidegger C, Knauf W, Trenn G, Kronawitter U, vanLunzen J, Arasteh K, Herbst H: HIV-related Non-Hodgkin's Lymphoma: CHOP-induction therapy and interferon- $\alpha$ - $2 \mathrm{~b} /$ zidovudine maintenance therapy. Leuk Lymph 1998;29:103-118.

17 Sparano JA, Hu X, Wiernik PH, Sarta C, Reddy DM, Hanau L, Henry DH: Opportunistic infection and immunologic function in patients with human immunodeficiency virus-associated non-Hodgkin's lymphoma treated with chemotherapy. J Natl Cancer Inst 1997;89:301-307.

18 Vaccher E, Spina M, Talamini R, Lucenti A, Errante D, Tavio M, Nasti G, Santarossa S, Tirelli U: Concomitant CHOP chemotherapy and highly active antiretroviral therapy (HAART) in patients (pts) with HIV-related Non-Hodgkin's Lymphoma (HIVNHL). Proc ASCO 1998;47a(abstr 184).

19 Tan B, Ratner L: The use of new antiretroviral therapy in combination with chemotherapy. Curr Opin Oncol 1997;9:455-464.

20 Mainman M, Fruchter RG, Clark M, Arrastia CD Matthews R, Gates EJ: Cervical cancer as an AIDSdefining illness. Obstet Gynecol 1997;89:76-80.

21 Goedert JJ, Cote TR, Virgo P, Scoppa SM, Kingma DW, Gail MH, Jaffe ES, Biggar RJ, for the AIDS Cancer Match Study group: Spectrum of AIDSassociated malignant disorders. Lancet 1998;351: 1833-1839.

22 Volm MD, von Roenn JH: Non-AIDS-defining malignancies in patientes with HIV-infection. Curr Opin Oncol 1996;8:386-391.

23 Hentrich M, Brack N, Schmid P, Schuster T, Clemm $\mathrm{Ch}$, Hartenstein R: Testicular germ cell tumors in patients with human immunodeficiency virus infection. Cancer 1996;77:2109-2116.

24 Peddada AV, Smith DE, Rao AR, Frost DB, Kagan AR: Chemotherapy and low-dose radiotherapy in the treatment of HIV-infected patients with carcinoma of the anal canal. Int J Rad Oncol Biol Phys 1997;37:1101-1105. 